\title{
Partitioning of Any Infinite Set with the Aid of Non-Surjective Injective Maps and the Study of a Remarkable Semigroup
}

\author{
Charif Harrafa 1 (1) \\ Ecole Hassania des Travaux Publics, Casablanca, Morocco \\ Email: charifeva@gmail.com
}

How to cite this paper: Harrafa, C. (2020) Partitioning of Any Infinite Set with the Aid of Non-Surjective Injective Maps and the Study of a Remarkable Semigroup. Open Journal of Discrete Mathematics, 10, 74-88. https://doi.org/10.4236/ojdm.2020.103008

Received: April 21, 2020

Accepted: July 6, 2020

Published: July 9, 2020

Copyright ( 2020 by author(s) and Scientific Research Publishing Inc. This work is licensed under the Creative Commons Attribution International License (CC BY 4.0).

http://creativecommons.org/licenses/by/4.0/

\begin{abstract}
In this article, we will present a particularly remarkable partitioning method of any infinite set with the aid of non-surjective injective maps. The non-surjective injective maps from an infinite set to itself constitute a semigroup for the law of composition bundled with certain properties allowing us to prove the existence of remarkable elements. Not to mention a compatible equivalence relation that allows transferring the said law to the quotient set, which can be provided with a lattice structure. Finally, we will present the concept of Co-injectivity and some of its properties.
\end{abstract}

\section{Keywords}

Partitioning, Non-Surjective, Injective, Infinite Set, Fixed Points, Lattice Structure

\section{Introduction}

The concept of map in mathematics has a primordial role in understanding the links that exist between the different mathematical fields and structures. A map is binary relation over two sets that associates to every element of the first set $e x$ actly one element of the second set, sometimes with a specific property. For instance, a "map" is a "linear transformation" in linear algebra, a "continuous function" in topology, operators in analysis and representations in group theory, etc. In this article we will show how non-surjective injective maps allow to partitioning an infinite set in several ways.

\section{Part I}

\section{Proposition 1}

Let $E$, and $F$ be non-empty sets. If $f, g$ are two non-surjective injective maps 
from $E$ to $F$ and from $F$ to $E$ respectively, then:

- $\left(A, f(B), I_{f \circ g}\right)$ forms a partition of $F$.

- $\left(B, g(A), I_{g \circ f}\right)$ forms a partition of $E$.

where,

$$
A=\{y \in F \mid \forall x \in E, f(x) \neq y\} \text { and } B=\{x \in E \mid \forall y \in F, g(y) \neq x\}
$$

and $I_{f}, I_{g}$ representing the image sets under $f, g$ respectively.

\section{Proof}

Let $E$ and $F$ be two non-empty sets, and let $f$ and $g$ be two non-surjective injective maps from $E$ to $F$, and from $F$ to $E$ respectively. Since $f$ and $g$ are non-surjective, then the following sets:

$$
A=\{y \in F \mid \forall x \in E, f(x) \neq y\} \quad \text { and } \quad B=\{x \in E \mid \forall y \in F, g(y) \neq x\} \quad \text { are }
$$
non-empty.

Also, obviously $E=B \cup I_{g}$ such as $B \cap I_{g}=\varnothing$ as follows from the definition of $I_{g}$.

For any such map $f$ from $E$ to $F$ :

$$
I_{f}=f(E)=f\left(B \cup I_{g}\right)=f(B) \cup f\left(I_{g}\right)=f(B) \cup I_{f \circ g}
$$

So $F=A \cup f(B) \cup I_{f \circ g}$

Since $f$ is an injective map then:

$$
f\left(B \cap I_{g}\right)=f(B) \cap I_{f \circ g}=f(\varnothing)=\varnothing
$$

Therefore, $\left(A, f(B), I_{f \circ g}\right)$ forms a partition of $F$.

In analogy to the map $g$ from $F$ to $E,\left(B, g(A), I_{g \circ f}\right)$ forms a partition of $E$.

\section{Note 1}

This process could be applied repeatedly, and for each iteration, finer partitions of the sets $E$ and $F$ respectively will be obtained, e.g. after $2^{\text {nd }}$ iteration we have:

- $E=B \cup g(A) \cup(g \circ f)(B) \cup I_{g \circ f \circ g}$

- $F=A \cup f(B) \cup(f \circ g)(A) \cup I_{f \circ g \circ f}$

In case that $f$ and $g$ are (two) (2) different non-surjective injective maps from an infinite set $E$ to itself, we can compose either by $f$ or by $g$, or by both indefinitely. Thus several partition classes of $E$ can obtained, for example after a second ( ${ }^{\text {nd }}$ ) iteration

- $E=A_{f} \cup f\left(A_{f}\right) \cup f^{2}\left(A_{f}\right) \cup I_{f^{3}}$

- $E=A_{g} \cup g\left(A_{g}\right) \cup g^{2}\left(A_{g}\right) \cup I_{g^{3}}$

- $E=A_{f} \cup f\left(A_{g}\right) \cup(f \circ g)\left(A_{f}\right) \cup I_{f \circ g \circ f}$

- $E=A_{g} \cup g\left(A_{f}\right) \cup(g \circ f)\left(A_{g}\right) \cup I_{g \circ f \circ g}$

- $E=A_{f} \cup f\left(A_{g}\right) \cup(f \circ g)\left(A_{g}\right) \cup I_{f \circ g^{2}}$

- $E=A_{g} \cup g\left(A_{f}\right) \cup(g \circ f)\left(A_{f}\right) \cup I_{g \circ f^{2}}$

where,

$$
A_{f}=\{y \in F \mid \forall x \in E, f(x) \neq y\} \text { and } A_{g}=\{x \in E \mid \forall y \in F, g(y) \neq x\}
$$




\section{Example 1}

If $E=F=\mathbb{N}$ i.e. the set of natural numbers, $f$ and $g$ are two maps from $\mathbb{N}$ to itself (i.e. $f$ and $g$ are non-surjective injective) and defined by: $\mathbb{\nabla}$

- $\forall n \in \mathbb{N}, f(n)=2 n$

- $\forall n \in \mathbb{N}, g(n)=2 n+1$

Knowing that $A_{f}=2 \mathbb{N}+1$ and $A_{g}=2 \mathbb{N}$ then:

- $f\left(A_{g}\right)=4 \mathbb{N}$

- $g\left(A_{f}\right)=4 \mathbb{N}+3$

- $(f \circ g)\left(A_{f}\right)=8 \mathbb{N}+6$

- $(g \circ f)\left(A_{g}\right)=8 \mathbb{N}+1$

- $(f \circ g \circ f)(\mathbb{N})=8 \mathbb{N}+2$

- $(g \circ f \circ g)(\mathbb{N})=8 \mathbb{N}+5$

Therefore, we can partition the set $\mathbb{N}$ to the second $\left(2^{\text {nd }}\right)$ order by $f$ and $g$ such as:

$$
\begin{aligned}
& \mathbb{N}=(2 \mathbb{N}+1) \cup(4 \mathbb{N}) \cup(8 \mathbb{N}+6) \cup(8 \mathbb{N}+2) \\
& \mathbb{N}=(2 \mathbb{N}) \cup(4 \mathbb{N}+3) \cup(8 \mathbb{N}+1) \cup(8 \mathbb{N}+5)
\end{aligned}
$$

\subsection{Remarkable Partition}

\section{Proposition 2}

Let $E$ be an infinite set, $\mathbb{N}$ be the set of natural numbers, and $f$ be a nonsurjective injective map from $E$ to itself, such that:

$$
A_{f}=\{y \in E \mid \forall x \in E, f(x) \neq y\}
$$

Then,

$$
\forall n \in \mathbb{N}, E=\left[\bigcup_{i=0}^{i=n} f^{i}\left(A_{f}\right)\right] \cup I_{f^{n+1}}
$$

where, $f^{i}\left(A_{f}\right)=\underbrace{f \circ f \circ \cdots \circ f\left(A_{f}\right)}_{i \text { times }}$

\section{Proof}

By induction:

For $n=0$, we have, by definition $E=A_{f} \cup I_{f}$, and for $n=1$ the proposition 1 states that, if $E=F$ and $f=g$ then, $E=A_{f} \cup f\left(A_{f}\right) \cup I_{f^{2}}$ Suppose that the said property is true for $n$. Then, by composing by $f$, we get:

$$
I_{f}=f(E)=\left[\bigcup_{i=0}^{i=n} f^{i+1}\left(A_{f}\right)\right] \cup I_{f^{n+2}}=\left[\bigcup_{i=1}^{i=n+1} f^{i}\left(A_{f}\right)\right] \cup I_{f^{n+2}}
$$

Then,

$$
E=A_{f} \cup\left[\bigcup_{i=1}^{i=n+1} f^{i}\left(A_{f}\right)\right] \cup I_{f^{n+2}}=\left[\bigcup_{i=0}^{i=n+1} f^{i}\left(A_{f}\right)\right] \cup I_{f^{n+2}}
$$

Therefore,

$$
\forall n \in \mathbb{N}, E=\left[\bigcup_{i=0}^{i=n} f^{i}\left(A_{f}\right)\right] \cup I_{f^{n+1}}
$$




\section{Note 2}

For all non-surjective injective maps $f$ from an infinite set $E$ to itself,

$$
\forall n \in \mathbb{N}, A_{f^{n+1}}=\bigcup_{i=0}^{i=n} f^{i}\left(A_{f}\right)
$$

\section{Definition 1}

Let $f$ be a map from no-empty set $E$ to itself,

- $x \in E$ is a fixed point of $f$ if, $f(x)=x$.

- $A \subset E$ is a fixed point set of $f$ if, $f(A)=A$.

\section{Proposition 3}

For all non-surjective injective maps ffrom an infinite set $E$ to itself, $\forall n \in \mathbb{N}$, $f^{n}\left(A_{f}\right)$ contains no fixed points of $f$.

\section{Proof}

By induction:

For $n=0$, let $x \in A_{f}$, and by definition $\forall y \in E, f(y) \neq x$, particularly for $y=x$ so $f(x) \neq x$ then $A_{f}$ contains no fixed points of $f$. Suppose that the aforementioned property is true for $n$, let $x \in f^{n+1}\left(A_{f}\right)=f\left(f^{n}\left(A_{f}\right)\right)$, then $\exists y \in f^{n}\left(A_{f}\right)$ such that $x=f(y)$, we have $f(y) \neq y$ (by inductive hypothesis), since $f$ is injective then $f(f(y)) \neq f(y)$ so $f(x) \neq x$, then $\forall x \in f^{n+1}\left(A_{f}\right)$, $f(x) \neq x$. Therefore, $\forall n \in \mathbb{N}, f^{n}\left(A_{f}\right)$ contains no fixed points of $f$.

\section{Note 3}

Let $E$ be an infinite set, and $f$ be a non-surjective injective map from $E$ to itself, we define the following:

$$
\text { Fix }_{f}=\{x \in E \mid f(x)=x\} \text { and } S t_{f}(E)=\{A \subset E \mid f(A)=A\}
$$

- $\forall n \in \mathbb{N}$, Fix $_{f} \cap f^{n}\left(A_{f}\right)=\varnothing$

- $\forall n \in \mathbb{N}^{*}$, Fix $_{f} \subseteq$ Fix $_{f^{n}}$

- $\forall n \in \mathbb{N}^{*}$, Fix $_{f^{n}} \subseteq$ Fix $_{f^{2 n}}$

- $\forall p \in \mathbb{N}, \forall n \in \mathbb{N}^{*}, f^{p}\left(\right.$ Fix $\left._{f^{n}}\right)=$ Fix $_{f^{n}}$

- For all $f$ non-surjective injective maps from an infinite set $E$ to itself,

$$
\mathrm{Fix}_{f} \in S t_{f}(E)
$$

\section{Proposition 4}

For all $f$ non-surjective injective maps from $E$ to itself,

$$
\forall A \in S t_{f}(E), \forall n \in \mathbb{N}, f^{n}\left(A_{f}\right) \cap A=\varnothing
$$

\section{Proof}

Let $A \in S t_{f}(E)$, by induction, For $n=0$, we have, by definition $A_{f} \cap I_{f}=\varnothing$ then $A_{f} \cap A=A_{f} \cap f(A)=\varnothing \bigotimes$ Suppose that the said property is true for $n \in \mathbb{N}$, let $x \in f^{n+1}\left(A_{f}\right) \cap A$, then, $\exists y \in f^{n}\left(A_{f}\right), x=f(y)$ and $\exists y_{0} \in A, x=f\left(y_{0}\right)$. Since $f$ is injective so $y=y_{0} \in f^{n}\left(A_{f}\right) \cap A$, which contradicts the inductive hypothesis. Then,

$$
f^{n+1}\left(A_{f}\right) \cap A=\varnothing .
$$


Therefore, $\forall A \in S t_{f}(E), \forall n \in \mathbb{N}, f^{n}\left(A_{f}\right) \cap A=\varnothing$

\section{Lemma 1 (Generalization)}

$$
\forall p \in \mathbb{N}^{*}, \forall A \in S t_{f^{p}}(E), \forall n \in \mathbb{N}, f^{n}\left(A_{f}\right) \cap A=\varnothing
$$

\section{Proof}

By complete (strong) induction,

Let $p \in \mathbb{N}^{*}$, and $A \in S t_{f^{p}}(E)=\left\{A \subset E \mid f^{p}(A)=A\right\}:$ For $n=0$, we have, by definition $A_{f} \cap I_{f}=\varnothing$, since $\exists p \in \mathbb{N}^{*}, I_{f^{p}} \subseteq I_{f}$, then:

$A_{f} \cap A=A_{f} \cap f^{p}(A)=\varnothing$

Suppose that the said property is true for all $i \in\{1, \cdots, n\}$, let $x \in f^{n+1}\left(A_{f}\right) \cap A$, then, $\exists y \in A_{f}, x=f^{n+1}(y)$ and $\exists y_{0} \in A, x=f^{p}\left(y_{0}\right)$. Since $f$ is injective then,

- $f^{n-p+1}(y)=y_{0}$, if $n+1>p$, which contradicts the inductive hypothesis, because $i=n-p+1 \in\{1, \cdots, n\}$

- $y=f^{p-n-1}\left(y_{0}\right)$, if $p>n+1$, which contradicts $A_{f} \cap I_{f^{p-n-1}}=\varnothing$

- $y=y_{0}$, if $n+1=p$, which contradicts $A_{f} \cap A=\varnothing$

Then,

$$
f^{n+1}\left(A_{f}\right) \cap A=\varnothing
$$

Therefore,

$$
\forall p \in \mathbb{N}^{*}, \forall A \in S t_{f^{p}}(E), \forall n \in \mathbb{N}, f^{n}\left(A_{f}\right) \cap A=\varnothing
$$

QED

For all $n \in \mathbb{N}$, and for all $f$ non-surjective injective maps from an infinite set $E$ to itself, we define the following:

- $S_{f^{n}}(E)=\left\{A \subset E \mid \exists p \in\{1, \cdots, n+1\}, f^{p}(A)=A\right\}$

- $S_{f^{\infty}}(E)=\left\{A \subset E \mid \exists p \in \mathbb{N}, f^{p}(A)=A\right\}$

- $S_{f^{n}}=\left\{x \in A \mid A \in S_{f^{n}}(E)\right\}$

- $S_{f^{\infty}}=\left\{x \in A \mid A \in S_{f^{\infty}}(E)\right\}$

\section{Theorem 1}

Let $E$ be an infinite set, and $f$ be a non-surjective injective map from $E$ to itself, then:

$$
E=\left[\bigcup_{n \in \mathbb{N}} f^{n}\left(A_{f}\right)\right] \cup S_{f^{\infty}}
$$

\section{Proof}

Note that for $n=0, S_{f^{0}}(E)=S t_{f}(E)$, The sequence of subsets of $E,\left(S_{f^{n}}\right)_{n \in \mathbb{N}}$ is increasing by inclusion, so it is convergent, the limit is: $\bigcup_{n \in \mathbb{N}} S_{f^{n}}=S_{f^{\infty}}$

We have already established that,

$$
\forall n \in \mathbb{N}, E=\left[\bigcup_{i=0}^{i=n} f^{i}\left(A_{f}\right)\right] \cup I_{f^{n+1}}
$$

Otherly, according to the Lemma $1, \forall n \in \mathbb{N}, E=\left[\bigcup_{i=0}^{i=n} f^{i}\left(A_{f}\right)\right] \cap S_{f^{n}}=\varnothing$

Let's define the following, $\forall n \in \mathbb{N}, \widehat{I_{f^{n+1}}}=I_{f^{n+1}} \backslash S_{f^{n}}$ the sequence of subsets 
of $\left(\widehat{I_{f^{n+1}}}\right)_{n \in \mathbb{N}}$ is decreasing by inclusion then, it is convergent [1], the limit is:

$$
\bigcap_{n \in \mathbb{N}} \widehat{I_{f^{n+1}}}=\varnothing
$$

Because, let $x \in \bigcap_{n \in \mathbb{N}} \widehat{I_{f^{n+1}}}$, then:

$$
\begin{gathered}
x \in \bigcap_{n \in \mathbb{N}} I_{f^{n+1}} \backslash S_{f^{n}} \Leftrightarrow \forall n \in \mathbb{N}, x \in I_{f^{n+1}} \backslash S_{f^{n}} \Leftrightarrow \forall n \in \mathbb{N}, x \in I_{f^{n+1}} \text { and } x \in \overline{S_{f^{n}}} \\
\Leftrightarrow \forall n \in \mathbb{N}, x \in I_{f^{n+1}} \text { and } \forall n \in \mathbb{N}, x \in \overline{S_{f^{n}}} \Leftrightarrow x \in \bigcap_{n \in \mathbb{N}} I_{f^{n+1}} \text { and } x \in \bigcap_{n \in \mathbb{N}} \overline{S_{f^{n}}} \\
\Leftrightarrow x \in \bigcap_{n \in \mathbb{N}} I_{f^{n+1}} \text { and } x \in \overline{\bigcup_{n \in \mathbb{N}} S_{f^{n}}} \Leftrightarrow x \in \bigcap_{n \in \mathbb{N}} I_{f^{n+1}} \text { and } x \in \overline{S_{f^{\infty}}} \\
\Leftrightarrow x \in\left[\bigcap_{n \in \mathbb{N}} I_{f^{n+1}}\right] \backslash S_{f^{\infty}}
\end{gathered}
$$

On the other hand, the sequence of subsets of $E,\left(I_{f^{n+1}}\right)_{n \in \mathbb{N}}$ is strictly decreasing by inclusion, then it is convergent and the limit is $H=\bigcap_{n \in \mathbb{N}} I_{f^{n+1}}$, and since $f$ is injective, so $f(H)=H$ then $f$ is bijective from $H$ to itself, and $H \in S t_{f}(E) \subset S_{f^{\infty}}(E)$.

$$
\forall n \in \mathbb{N}, E=\left[\bigcup_{i=0}^{i=n} f^{i}\left(A_{f}\right)\right] \bigcup \widehat{I_{f^{n+1}}} \cup S_{f^{n}}
$$

Therefore,

$$
E=\left[\bigcup_{n \in \mathbb{N}} f^{n}\left(A_{f}\right)\right] \cup S_{f^{\infty}}
$$

N.B. If $S_{f^{\infty}}=\varnothing$, then we get:

$$
E=\bigcup_{n \in \mathbb{N}} f^{n}\left(A_{f}\right)
$$

\section{Example 2}

QED

$\mathbb{R}$ is the set of real numbers, and $P(\mathbb{R})$ is the set of subsets of $\mathbb{R}$.

Let $f$ be a map from $\mathbb{R}$ to itself, defined by:

$$
f(x)= \begin{cases}x+1, & \text { if } x \geq 0 \\ x, & \text { if } x<0\end{cases}
$$

Therefore, $f$ is injective non-surjective from $\mathbb{R}$ to itself, because $f$ is injective and $A_{f}=[0,1[$.

where,

$$
\forall n \in \mathbb{N}^{*}, f^{n}(x)= \begin{cases}x+n, & \text { if } x \geq 0 \\ x, & \text { if } x<0\end{cases}
$$

We have, $f\left(A_{f}\right)=\left[f(0), f(1)\left[=\left[1,2\left[\subset \mathbb{R}_{+}\right.\right.\right.\right.$since $f$ is increasing Therefore,

$$
\forall n \in \mathbb{N}^{*}, f^{n}\left(A_{f}\right)=[n, n+1[
$$

- $\forall n \in \mathbb{N}, S_{f^{n}}=S_{f^{\infty}}=\mathbb{R}_{-}^{*}$

- $\forall n \in \mathbb{N}, S_{f^{n}}(E)=S_{f^{\infty}}(E)=P\left(\mathbb{R}_{-}^{*}\right)$ 
According to the theorem 1, we can write:

$$
\mathbb{R}=\mathbb{R}_{-}^{*} \cup\left[\bigcup_{n \in \mathbb{N}}[n, n+1[]\right.
$$

\section{Example 3}

\section{Remark}

If $h$ is an affine map from $\mathbb{R}$ to $\mathbb{R}$, so that $h(x)=a x+b / a, b \in \mathbb{R}$ and $a \neq 0$, then:

$$
\forall n \in \mathbb{N}^{*}, \forall x \in \mathbb{R}, h^{n}(x)=a^{n} x+b\left(1+a+\cdots+a^{n-1}\right)
$$

If $a \neq 1$, then:

$$
\forall n \in \mathbb{N}^{*}, \forall x \in \mathbb{R}, h^{n}(x)=a^{n} x+b\left(\frac{1-a^{n}}{1-a}\right)
$$

Let $f$ be a map defined from $E=[0,4]$ to itself by:

$$
\forall x \in[0,4], f(x)= \begin{cases}-x+1, & \text { if } x \in[0,1] \\ x+1, & \text { if } x \in] 1,2] \\ \frac{1}{2} x+2, & \text { if } x \in[2,4]\end{cases}
$$

$f$ is non-surjective injective map from $E$ to itself, so that:

- $\left.\left.A_{f}=\right] 1,2\right]$

- Fix $_{f}=\{4\}$

- The set $A=[0,1] \subset E$, fulfills the condition $f(A)=A$

- We have $\left.\left.\left.\left.\left.\left.\left.\left.\left.\left.A_{f}=\right] 1,2\right], f\left(A_{f}\right)=f(] 1,2\right]\right)=\right] 2,3\right] \subset[2,4], \quad f(] 2,3\right]\right)=\right] 3, \frac{7}{2}\right]$, ...

- $S_{f^{\infty}}=\{4\} \cup[0,1]$

The restriction of $f$ to $[2,4]$ is an affine function such as $a=\frac{1}{2}$, and $b=2$, then:

$$
\forall n \in \mathbb{N}^{*}, f^{n}(x)=\frac{1}{2^{n}} x+2\left(1+\frac{1}{2}+\cdots+\frac{1}{2^{n-1}}\right)
$$

We have: $f^{n}(2)=4-\frac{1}{2^{n-1}}$, and $f^{n}(3)=4-\frac{1}{2^{n}}$,

According to Theorem 1:

$$
\left.\left.[0,4]=\left\{[1,2] \bigcup_{n \in \mathbb{N}}\right] 4-\frac{1}{2^{n-1}}, 4-\frac{1}{2^{n}}\right]\right\} \cup\{4\} \cup[0,1]
$$

\section{Example 4}

Let $f$ be a map defined from $E=[0,3]$ to itself by:

$$
\forall x \in[0,3], f(x)= \begin{cases}x+2, & \text { if } x \in[0,1] \\ \frac{1}{2} x+1, & \text { if } x \in] 1,2[ \\ x-2, & \text { if } x \in[2,3]\end{cases}
$$


$f$ is non-surjective injective from $E$ to itself, so that:

- $\left.\left.A_{f}=\right] 1, \frac{3}{2}\right]$

- Fix $_{f}=\varnothing$

- The set $A=[0,1] \subset E$, fulfills the condition $f(A) \neq A$, and $f^{2}(A)=A$

- The set $B=[2,3] \subset E$, fulfills the condition $f(B) \neq B$, and $f^{2}(B)=B$

- $S_{f^{\infty}}=[0,1] \cup[2,3]$

We have,

- $\forall x \in] 1,2\left[, \forall n \in \mathbb{N}^{*}, f^{n}(x)=\frac{1}{2^{n}} x+2\left(1-\frac{1}{2^{n}}\right)\right.$

- $\lim _{x \rightarrow 1^{-}} f^{n}(x)=2-\frac{1}{2^{n}}$ and $f^{n}\left(\frac{3}{2}\right)=2-\frac{1}{2^{n+1}}$

Therefore, according to Theorem 1:

$$
\left.\left.[0,3]=\left\{\bigcup_{n \in \mathbb{N}}\right] 2-\frac{1}{2^{n}}, 2-\frac{1}{2^{n+1}}\right]\right\} \bigcup[0,1] \cup[2,3]
$$

\section{Corollary 1}

Let $f, g$ be non-surjective injective maps from an infinite set $E$ to itself such as $S_{f^{\infty}}=\overline{S_{g^{\infty}}}$, then:

$$
E=\bigcup_{n \in \mathbb{N}} B_{n}
$$

where, $\forall n \in \mathbb{N}, B_{n}=f^{n}\left(A_{f}\right) \cup g^{n}\left(A_{g}\right)$

Therefore, $\left(B_{n}\right)_{n \in \mathbb{N}}$ forms a partition of $E$.

\section{Definition 2}

- Let $E$ be an infinite set, we write: $\operatorname{Injns}(E)$ as being the set of non-surjective injective maps from $E$ to itself.

- $\operatorname{Injns}(E, F)$ as the set of non-surjective injective maps from a set $E$ to a set $F$, that being said, $E$ and $F$ are supposed to be non-empty and $|E| \leq|F|(|E|$ is the cardinal of $E$ ).

\section{Properties}

1) $(\operatorname{Injns}(E), \circ)$ is a semigroup, because the composite of 2 (two) injective maps $f$ and $g$ is injective and $I_{f \circ g} \subset I_{f}$, so $\forall f, g \in \operatorname{Injns}(E), f \circ g \in \operatorname{Injns}(E)$.

2) $\forall f, g \in \operatorname{Injns}(E), A_{f \circ g}=A_{f} \cup f\left(A_{g}\right),\left(A_{f}, f\left(A_{g}\right), I_{f \circ g}\right)$ is, indeed, a partition of $E$ and $E=A_{f \circ g} \cup I_{f \circ g}$, because $f \circ g \in \operatorname{Injns}(E)$.

3) There is no idempotent element for the law of composition in $\operatorname{Injns}(E)$ and if such an element exists, then $f^{2}=f$ and since $f$ is injective, then $f=I_{d}$ which is contradictory, because, $f \in \operatorname{Injns}(E)$.

4)Let $f \in \operatorname{Injns}(E)$, and assuming that $f$ is a map from $E$ to itself such as: $\tilde{f}(x)= \begin{cases}x, & \text { if } x \in A_{f} \\ f(x), & \text { if } x \in I_{f}\end{cases}$

5) We have, $\forall f \in \operatorname{Injns}(E), \tilde{f} \in \operatorname{Injns}(E)$ knowing that $I_{\tilde{f}}=A_{f} \cup I_{f^{2}}$ and $A_{\tilde{f}}=f\left(A_{f}\right)$

6) Let $f \in \operatorname{Injns}(E)$, we have, $\tilde{f}\left(A_{\tilde{f}}\right)=\tilde{f}\left(f\left(A_{f}\right)\right)=f^{2}\left(A_{f}\right)$, and 


$$
I_{\tilde{f}^{2}}=\tilde{f}\left(I_{\tilde{f}}\right)=\tilde{f}\left(A_{f} \cup I_{f^{2}}\right)=\tilde{f}\left(A_{f}\right) \cup \tilde{f}\left(I_{f^{2}}\right)=A_{f} \cup I_{f^{3}}
$$

Then,

$$
E=A_{\tilde{f}} \cup \tilde{f}\left(A_{\tilde{f}}\right) \cup I_{\tilde{f}^{2}}=A_{f} \cup f\left(A_{f}\right) \cup f^{2}\left(A_{f}\right) \cup I_{f^{3}}
$$

Therefore, $\tilde{f}$ allow us to reduce order of iteration.

7) $\forall f \in \operatorname{Injns}(E)$,

- $A_{\tilde{f} \circ f}=A_{\tilde{f}} \cup \tilde{f}\left(A_{f}\right)=A_{f} \cup f\left(A_{f}\right)=A_{f^{2}}$, then $I_{\tilde{f} \circ f}=I_{f^{2}}$

- $A_{f \circ \tilde{f}}=A_{f} \cup f\left(A_{\tilde{f}}\right)=A_{f} \cup f^{2}\left(A_{f}\right)$, then, $I_{f \circ \tilde{f}}=f\left(A_{f}\right) \cup I_{f^{3}}$

- $A_{\tilde{f}}=\tilde{f}\left(A_{\tilde{f}}\right)=f^{2}\left(A_{f}\right)$, then $I_{\tilde{f}}=A_{f} \cup f\left(A_{f}\right) \cup I_{f^{3}}$

8) Let $f \in \operatorname{Injns}(E, F)$ and $g \in \operatorname{Injns}(F, E)$, so:

$$
f \circ g \in \operatorname{Injns}(F) \text { and } g \circ f \in \operatorname{Injns}(E)
$$

\subsection{Equivalence Relations}

\section{Example 5}

Let $f, g \in \operatorname{Injns}(E)$, we define the binary relation $R$ on $\operatorname{Injns}(E)$, by:

$$
f R g \Leftrightarrow A_{f}=A_{f} \Leftrightarrow I_{f}=I_{g}
$$

$R$ is, indeed, an equivalence relation, because:

- $R$ is reflexive, $f R f, \forall f \in \operatorname{Injns}(E)$

- $R$ is symmetric, $f R g \Leftrightarrow A_{f}=A_{g} \Leftrightarrow g R f, \forall f, g \in \operatorname{Injns}(E)$

- $R$ is transitive, $\forall f, g$ and $h \in \operatorname{Injns}(E), f R g$ and $g R h \Leftrightarrow A_{f}=A_{g}$ and $A_{g}=A_{h} \Leftrightarrow A_{f}=A_{h} \Leftrightarrow f R h$

\section{Example 6}

Let $f, g \in \operatorname{Injns}(E)$, we define the binary relation $R$ on $\operatorname{Injns}(E)$, by:

$$
\forall f, g \in \operatorname{Injns}(E): f R g \Leftrightarrow \forall n \in \mathbb{N}^{*}, I_{f^{n}}=I_{g^{n}}
$$

$R$ is, indeed, an equivalence relation, because:

- $R$ is reflexive, $f R f, \forall f \in \operatorname{Injns}(E)$

- $R$ is symmetric, $f R g \Leftrightarrow \forall n \in \mathbb{N}^{*}, I_{f^{n}}=I_{g^{n}} \Leftrightarrow g R f, \forall f, g \in \operatorname{Injns}(E)$

- $R$ is transitive, $f, g$ and $h \in \operatorname{Injns}(E), f R g$ and

$$
\begin{aligned}
& g R h \Leftrightarrow \forall n \in \mathbb{N}^{*}, I_{f^{n}}=I_{g^{n}} \text { and } \\
& \forall n \in \mathbb{N}^{*}, I_{g^{n}}=I_{h^{n}} \Leftrightarrow \forall n \in \mathbb{N}^{*}, I_{f^{n}}=I_{h^{n}} \Leftrightarrow f R h
\end{aligned}
$$

\section{Example 7}

$$
\forall f, g \in \operatorname{Injns}(E), f R g \Leftrightarrow f\left(A_{f}\right)=g\left(A_{g}\right)
$$

\section{Example 8}

$$
\forall f, g \in \operatorname{Injns}(E), f R g \Leftrightarrow S_{f^{\infty}}=S_{g^{\infty}} \Leftrightarrow \bigcup_{n \in \mathbb{N}} f^{n}\left(A_{f}\right)=\bigcup_{n \in \mathbb{N}} g^{n}\left(A_{g}\right)
$$

$\bigcup_{n \in \mathbb{N}} f^{n}\left(A_{f}\right)$ : as being the $f$-semicoverage of a set $E$.

\section{Part II}

Let $E$ be an infinite set, $f \in \operatorname{Injns}(E)$, and $\bar{f}=\left\{g \in \operatorname{Injns}(E) \mid I_{g} \cap I_{f} \neq \varnothing\right\}$ 
We get the following:

- $\forall f \in \operatorname{Injns}(E), \forall n \in \mathbb{N}^{*}, f^{n} \in \bar{f}$

- $\forall f, g \in \operatorname{Injns}(E): I_{f} \subset I_{g} \Rightarrow \bar{f} \subset \bar{g}$

Let $h \in \bar{f}$ i.e. $I_{f} \cap I_{h} \neq \varnothing$ let $x \in I_{h} \cap I_{f}$, so $x \in I_{h}$ and $x \in I_{f} \subset I_{g}$, so $x \in I_{h}$ and $x \in I_{g}$, so $x \in I_{h} \cap I_{g}$ so $I_{h} \cap I_{g} \neq \varnothing$, then $h \in \bar{g}$ therefore $\bar{f} \subset \bar{g}$

\section{Theorem 2}

Let $E$ be an infinite set, then there exists a non-surjective injective map $\psi$ from $E$ to itself, so that for any such non-surjective injective map $\phi$ from $E$ to itself, we have:

$$
I_{\psi} \cap I_{\phi} \neq \varnothing
$$

\section{Proof}

By contradiction, let's suppose that for all $\psi$ non-surjective injective maps from $E$ to itself, there exists a map $\phi_{\psi} \in \operatorname{Injns}(E)$ such as $I_{\psi} \cap I_{\phi_{\psi}}=\varnothing$ so that $I_{\phi_{\psi}} \subseteq A_{\psi}$. Additionally $E$ is an infinite set, so $E$ is equipotential [2] to $E \backslash\{a\}, \forall a \in E$. Considering this bijection $f$ as a map from $E$ to itself, then $f \in \operatorname{Injns}(E)$, and $A_{f}=\{a\}$, according to all of the above, there exists a map $f_{\phi} \in \operatorname{Injns}(E)$ such as $I_{f_{\phi}} \subseteq A f=\{a\}$, which contradicts the fact that $f_{\phi}$ injective.

QED

\section{Note 4}

- $\exists \psi \in \operatorname{Injns}(E)$, so that: $\forall \varphi \in \operatorname{Injns}(E), \psi^{-1}\left(I_{\varphi}\right) \neq \varnothing$

- If $\psi$ applies to the former theorem then: $\bar{\psi}=\operatorname{Injns}(E)$.

\section{Proposition 5}

$\forall f \in \operatorname{Injns}(E), \exists g \in \operatorname{Injns}(E) \backslash\{f\}$, so that $A_{f} \cap A_{g} \neq \varnothing$

\section{Proof}

By contradiction, assuming that exists a map $f \in \operatorname{Injns}(E)$, so that $\forall g \in \operatorname{Injns}(E) \backslash\{f\}, A_{f} \cap A_{g}=\varnothing$, then $\forall g \in \operatorname{Injns}(E) \backslash\{f\}, I_{f} \cup I_{g}=E$.

Let $g=f^{2}$, so $I_{f} \cup I_{g}=I_{f} \subsetneq E$ because $f \in \operatorname{Injns}(E)$ which is contradictory.

\section{Proposition 6}

$\forall f \in \operatorname{Injns}(E), \exists g \in \operatorname{Injns}(E) \backslash\{f\}$, such as $A_{f} \cap I_{g} \neq \varnothing$

\section{Proof}

By contradiction, assuming that exists a map $f \in \operatorname{Injns}(E)$, so that, $\forall g \in \operatorname{Injns}(E) \backslash\{f\}, A_{f} \cap I_{g}=\varnothing$ then $\forall g \in \operatorname{Injns}(E) \backslash\{f\}, I_{f} \cup A_{g}=E$. On the other hand, if $g=\tilde{f}, I_{f} \cup A_{g}=E$, additionally $A_{g}=f\left(A_{f}\right) \subset I_{f}$ so $I_{f} \cup A_{g}=I_{f}=E$ which is contradictory.

\section{Note 5}

We can define another composition law $T$ for $\operatorname{Injns}(E)$ so that,

$$
\forall f, g \in \operatorname{Injns}(E),(f T g)(x)=\left\{\begin{array}{l}
g(x), \text { if } x \in f^{-1}\left(I_{g}\right) \\
f(x), \text { if } x \in f^{-1}\left(A_{g}\right)
\end{array}\right.
$$

- $f T f=f, \forall f \in \operatorname{Injns}(E)$ then every element is idempotent under the law $T$. 
- $\forall f, g \in \operatorname{Injns}(E)$, if $I_{f} \cap I_{g}=\varnothing$, then $f T g=f$.

- $\forall f, g \in \operatorname{Injns}(E)$, if $I_{f} \subseteq I_{g}$, then $f T g=g$.

- $\forall f \in \operatorname{Injns}(E), \quad f T \tilde{f}=f$ and $\tilde{f} T f=\tilde{f}$.

- Generally, $\forall f, g \in \operatorname{Injns}(E), \quad f T g \neq g T f$.

\section{Part III}

\subsection{Study of the Quotient Set}

Let $E$ be an infinite set, and $f, g \in \operatorname{Injns}(E)$. We define the binary relation $R$ on $\operatorname{Injns}(E)$, by:

$$
f R g \Leftrightarrow A_{f} \text { and } A_{g} \text { have the same cardinal }
$$

The binary relation $R$ is reflexive, symmetric and transitive, so $R$ is an equivalence relation on $\operatorname{Injns}(E)$.

We define $C l(f)=\left\{g \in \operatorname{Injns}(E)|| A_{g}|=| A_{f} \mid\right\}$ the equivalence class of a $\operatorname{map} f$.

\section{Note 6}

- Let $f \in \operatorname{Injns}(E)$, as $A_{\tilde{f}}=f\left(A_{f}\right)$ so $A_{f}$ and $A_{\tilde{f}}$ have the same cardinal, because $f$ is injective then $\tilde{f} \in C l(f)$, therefore,

$$
\forall f \in \operatorname{Injns}(E), \tilde{f} \in C l(f)
$$

- Let $f, g \in \operatorname{Injns}(E)$, assuming that the cardinals of $A_{f}$ and $A_{f}$ are finite, and thus, $\left|A_{f \circ g}\right|=\left|A_{f} \cup f\left(A_{g}\right)\right|=\left|A_{f}\right|+\left|A_{g}\right|-\left|A_{f} \cap f\left(A_{g}\right)\right|$, and since $A_{f} \cap f\left(A_{g}\right)=\varnothing$, then: $\left|A_{f \circ g}\right|=\left|A_{f} \cup f\left(A_{g}\right)\right|=\left|A_{f}\right|+\left|A_{g}\right|$. Since the composition of two (2) maps $f$ and $g$ on Injns $(E)$ yields a disjoint union, i.e. $A_{f \circ g}=A_{f} \cup f\left(A_{g}\right)$, with $A_{f} \cap f\left(A_{g}\right)=\varnothing$, then we can extend the sum of the cardinals even for infinite sets, such as:

$$
\left|A_{f \circ g}\right|=\left|A_{f} \cup f\left(A_{g}\right)\right|=\left|A_{f}\right|+\left|A_{g}\right|=\sup \left\{\left|A_{f}\right|,\left|A_{g}\right|\right\}
$$

- For all maps $f, g, h$ and $t \in \operatorname{Injns}(E)$ so that $f R g$ and hRt i.e. $\left|A_{f}\right|=\left|A_{g}\right|$ and $\left|A_{h}\right|=\left|A_{t}\right|$. Since:

$$
\begin{aligned}
\left|A_{f \circ h}\right|=\sup \left\{\left|A_{f}\right|,\left|A_{g}\right|\right\} & =\sup \left\{\left|A_{g}\right|,\left|A_{t}\right|\right\}=\left|A_{g \circ t}\right|, \text { therefore, } \\
& f \circ g R h \circ t
\end{aligned}
$$

- The map's composition law is compatible under the equivalence relation $R$, then we can provide the quotient set $(\operatorname{Injns}(E) / R, *)$ with a structure of a semigroup.

- $\forall C l(f)$ and $C l(g) \in \operatorname{Injns}(E) / R: C l(f) * C l(g)=C l(f \circ g)$

\subsection{Partial Order}

Let $C l(f), C l(g) \in \operatorname{Injns}(E) / R$, define a binary relation on $\operatorname{Injns}(E) / R$ by:

$$
C l(f) \leq C l(g) \Leftrightarrow\left|A_{f}\right| \leq\left|A_{g}\right|
$$

- $\forall C l(f) \in \operatorname{Injns}(E) / R, C l(f) \leq C l(f)$

So $\leq$ is reflexive. 
- $\forall C l(f), C l(g) \in \operatorname{Injns}(E) \backslash R, \quad C l(f) \leq C l(g)$ and $C l(g) \leq C l(f) \Leftrightarrow$ $\left|A_{f}\right| \leq\left|A_{g}\right|$ and $\left|A_{g}\right| \leq\left|A_{f}\right| \Leftrightarrow\left|A_{f}\right|=\left|A_{g}\right|$ so $C l(f)=C l(g)$

So $\leq$ is asymmetric.

- $\forall C l(f), C l(g)$ and $C l(h) \in \operatorname{Injns}(E) / R: C l(f) \leq C l(g)$ and $C l(g) \leq C l(h) \Leftrightarrow\left|A_{f}\right| \leq\left|A_{g}\right|$ and $\left|A_{g}\right| \leq\left|A_{h}\right| \Rightarrow\left|A_{f}\right| \leq\left|A_{h}\right| \Rightarrow$ $C l(f) \leq C l(h)$

So $\leq$ is transitive.

Therefore, the relation $\leq$ is a partial order on $\operatorname{Injns}(E) / R$.

Note 7

- Since $\forall C l(f), C l(g) \in \operatorname{Injns}(E) / R, \quad C l(f) \leq C l(g)$ or $C l(g) \leq C l(f)$ then $\leq$ is a total partial order on $\operatorname{Injns}(E) / R$.

- The partial order on the semigroup $(\operatorname{Injns}(E) / R, *)$ is, indeed, compatible [3] with the equivalence class's composition law of composition *, then:

$$
\forall C l(f), C l(g) \text { and } C l(h) \in \operatorname{Injns}(E) / R \text {, }
$$

If $C l(f) \leq C l(g)$ then

$C l(f) * C l(h)=C l(f \circ h) \leq C l(g \circ h)=C l(g) * C l(h)$ and

$C l(h) * C l(f)=C l(h \circ f) \leq C l(h \circ g)=C l(h) * C l(g)$

- Injns $(E) / R$ is well-ordered, because any non-empty subset has a smallest element.

- $\operatorname{Injns}(E) / R$ is a lattice, because it is ordered and every pair of elements has both upper bound and lower bound [4].

- $\operatorname{Injns}(E) / R$ provided with the order relation has a minimal element $\mathrm{Cl}(f)$, so that $\left|A_{f}\right|=1$, and also maximal element $\mathrm{Cl}(g)$, so that $A_{g}$ has the same cardinality as $E$.

- If $E$ is an infinite countable set, the map $\varphi$ defined by:

$$
\varphi: \operatorname{Injns}(E) \rightarrow \mathbb{N}^{*} \cup\{\aleph 0\}=M, \forall f \in \operatorname{Injns}(E), \varphi(f)=\left|A_{f}\right|,
$$

where, $\aleph 0$ represents the cardinal of $\mathbb{N}$.

Considering the convention: $\forall n \in \mathbb{N}^{*}, n+\aleph 0=\aleph 0, \varphi$ is a morphism of semigroups of $(\operatorname{Injns}(E), \circ)$ on $(M,+)$.

$$
\forall f, g \in \operatorname{Injns}(E), \varphi(f \circ g)=\left|A_{f \circ g}\right|=\left|A_{f}\right|+\left|A_{g}\right|=\varphi(f)+\varphi(g)
$$

\section{Complement}

Let $f, g \in \operatorname{Injns}(E)$ so that $\left|A_{f}\right|<\left|A_{g}\right|$, with the assumption that $A_{f}$ and $A_{g}$ are considered finite, $I_{f}$ and $I_{g}$ are equipotential because, the map $\psi$ defined from $I_{f}$ to $I_{g}$ by:

$\forall x \in I_{f}, \psi(x)=\left(g \circ f^{-1}\right)(x)$ is bijective, where $f^{-1}$ is defined from $I_{f}$ to $E$, and for all $x \in I_{f}$, we associated its inputs by $f$, we have:

- $\forall x, y \in I_{f}, \psi(x)=\psi(y) \Rightarrow\left(g \circ f^{-1}\right)(x)=\left(g \circ f^{-1}\right)(y) \Rightarrow$ $g\left(f^{-1}(x)\right)=g\left(f^{-1}(y)\right) \Rightarrow f^{-1}(x)=f^{-1}(y) \Rightarrow x=y \quad$ (because both $g$ and $f^{-1}$ are injectives). Therefore $\psi$ is injective.

- On the other hand $\psi\left(I_{f}\right)=\left(g \circ f^{-1}\right)\left(I_{f}\right)=g\left(f^{-1}\left(I_{f}\right)\right)=g(E)=I_{g}$ so $\psi$ is surjective. 
Let $\varphi \in \operatorname{Injns}\left(A_{f}, A_{g}\right)$ so that the map $\theta$ is defined by:

$$
\theta(x)=\left\{\begin{array}{l}
\varphi(x), \text { if } x \in A_{f} \\
\psi(x), \text { if } x \in I_{f}
\end{array}\right.
$$

Belong to $\operatorname{Injns}(E)$ and $A_{\theta}=A_{\varphi}$.

\section{Note 8}

- If $g=f^{2}$ so $\forall x \in I_{f}, \psi(x)=f(x)$ so if $\varphi=f / A_{f}$ then we have, $\theta=f$, and if $\varphi=I_{d} / A_{f}$ so $\theta=\tilde{f}$

- We considered, previously, that, $A_{f}$ and $A_{g}$ are finite. That said, we can build the $\varphi$ map even in case that $A_{f}$ and $A_{g}$ are infinite and that $\left|A_{f}\right|=\left|A_{g}\right|$.

\section{Part IV}

\section{E Permutations Group Action}

Let $\sigma(E)$ be the permutations group of $E, f \in \operatorname{Injns}(E)$ and $\sigma \in \sigma(E)$, $f \circ \sigma \in \operatorname{Injns}(E)$, because, since $f$ and $\sigma$ are injective then $f \circ \sigma$ is injective and $\forall \sigma \in \sigma(E),(f \circ \sigma)(E)=f(\sigma(E))=f(E)=I_{f} \subsetneq E$ then $f \circ \sigma \quad$ is non-surjective.

where $\forall \sigma \in \sigma(E), I_{f \circ \sigma}=I_{f}$ then $A_{f \circ \sigma}=A_{f}$.

We have,

- $\otimes \forall \sigma_{1}, \sigma_{2} \in \sigma(E)$ and $f \in \operatorname{Injns}(E):\left(f \circ \sigma_{1}\right) \circ \sigma_{2}=f \circ\left(\sigma_{1} \circ \sigma_{2}\right)$

- $\forall f \in \operatorname{Injns}(E), f \circ I_{d}=f$

$$
\text { Let } \theta: \sigma(E) \times \operatorname{Injns}(E) \rightarrow \operatorname{Injns}(E)
$$

so that $\forall \sigma \in \sigma(E), \forall f \in \operatorname{Injns}(E), \quad \theta(\sigma, f)=f \circ \sigma$

Therefore, the $E$ permutations group operates on the rightmost on $\operatorname{Injns}(E)$.

\section{Note 9}

The relation $R$ defined on Injns $(E)$ by: $f R g \Leftrightarrow \exists \sigma \in \sigma(E)$ such as $g=f \circ \sigma$ is an equivalence relation, that is called Intransitivity relation [5].

\section{Proposition 7}

Let be, $g \in \operatorname{Injns}(E): \exists \sigma \in \sigma(E)$ so that $g=f \circ \sigma \Leftrightarrow I_{f}=I_{g}$.

Proof

$\Rightarrow$ ) If there exists $\sigma \in \sigma(E)$ so that $g=f \circ \sigma \Rightarrow I_{g}=I_{f \circ \sigma}=I_{f}$.

$\Leftarrow)$ If $I_{f}=I_{g}$, then the map $\sigma: E \rightarrow E$, so that $x \mapsto \sigma(x)=f^{-1}(g(x))$ is bijective, because:

- $\sigma(E)=f^{-1}(g(E))=E$ so $\sigma$ is surjective.

- $\forall x, y \in E: \sigma(x)=\sigma(y) \Rightarrow f^{-1}(g(x))=f^{-1}(g(y)) \Rightarrow g(x)=g(y)$ $\Rightarrow \quad x=y$ so $\sigma$ is injective.

- $\forall x \in E, f \circ \sigma(x)=f(\sigma(x))=g(x)$

Note 10

- The equivalence class (Intransitivity relation) of the element $f$ is called the orbit of $f, C l(f)=\{f \circ \sigma \mid \sigma \in \sigma(E)\}=\left\{g \in \operatorname{Injns}(E) \mid I_{g}=I_{f}\right\}$.

- The stabilizer of $f$ is: $\Delta f=\{\sigma \in \sigma(E) \mid f \circ \sigma=f\}=\left\{I_{d}\right\}$, i.e. the morphism associated with the said action is injective. 


\section{Part V}

Let $f, g \in \operatorname{Injns}(E)$.

Definition 3

$f$ and $g$ are said to be Co-injectives, if,

$$
I_{f} \cap I_{g} \neq \varnothing \text { and } \forall x, y \in E, f(x)=g(y) \Rightarrow x=y
$$

Let $\hat{f}=\{g \in \operatorname{Injns}(E) \mid g$ is Co-injective with $f\}$

We have $\hat{f} \neq \varnothing$ because $\forall f \in \operatorname{Injns}(E), I_{f} \cap I_{f} \neq \varnothing$ and $\forall x, y \in E$, $f(x)=f(y) \Rightarrow x=y \quad$ so $f$ is Co-injective with itself.

Therefore $\forall f \in \operatorname{Injns}(E), \quad f \in \hat{f}$, then, $\forall f \in \operatorname{Injns}(E): \hat{f} \neq \phi$

\section{Proposition 8}

Let $h \in \operatorname{Injns}(E), \quad \forall f, g \in \operatorname{Injns}(E)$ :

$f, g$ are Co-injectives $\Rightarrow h \circ f$ and $h \circ g$ are Co-injectives.

\section{Proof:}

Let $h \in \operatorname{Injns}(E)$, and $f, g \in \operatorname{Injns}(E)$ such as $f$ and $g$ are Co-injective. $I_{f} \cap I_{g} \neq \varnothing$, additionally $h\left(I_{f} \cap I_{g}\right)=I_{h \circ f} \cap I_{\text {hog }} \neq \varnothing \quad$ ( $h$ is injective).

Let $x, y \in E, h \circ f(x)=h \circ g(y) \Rightarrow f(x)=g(y) \Rightarrow x=y \quad$ (because $h$ is injective and $f, g$ are Co-injectives).

Therefore $h \circ f$ and $h \circ g$ are Co-injectives.

\section{Note 11}

For all $f, g \in \operatorname{Injns}(E)$, so that $f, g$ are Co-injectives, so: $\square$

- $f^{2}$ and $f \circ g$ are Co-injectives

- $\forall z \in I_{f} \cap I_{g}, \quad \exists ! x \in E$, so that, $z=f(x)=g(x)$

- $f^{-1}\left(I_{g}\right)=g^{-1}\left(I_{f}\right)$

- If, $I_{f}=I_{g}$, then $f=g \otimes$

- Let $h \in \operatorname{Injns}(E)$, if $f\left(I_{h}\right) \cap g\left(I_{h}\right) \neq \varnothing$, then $f \circ h$ and $g \circ h$ are Coinjectives.

\section{Proposition 9}

$\forall f, g \in \operatorname{Injns}(E)$, if $I_{f} \subsetneq I_{g}$, then $f, g$ are not Co-injective.

\section{Proof}

$\forall f, g \in \operatorname{Injns}(E)$, suppose that $I_{f} \subsetneq I_{g}$, then we have $I_{g}=I_{f} \cup\left(I_{g} \backslash I_{f}\right)$. If $f, g$ are Co-injectives, then $f^{-1}\left(I_{g}\right)=g^{-1}\left(I_{f}\right)=E$, which is contradictory because:

$$
E=g^{-1}\left(I_{f}\right) \cup g^{-1}\left(I_{g} \backslash I_{f}\right) \text {, and } g^{-1}\left(I_{g} \backslash I_{f}\right) \neq \varnothing, \text { so } g^{-1}\left(I_{f}\right) \subsetneq E .
$$

\section{Proposition 10}

Let $f, g \in \operatorname{Injns}(E)$, so that $f$ and $g$ are Co-injectives, $\forall h \in \operatorname{Injns}(E)$, if $g$ and $h$ are Co-injectives and $I_{f} \cap I_{h}=I_{f} \cap I_{g}$ then $f$ and $h$ are Co-injectives.

\section{Proof}

$I_{f} \cap I_{h} \neq \varnothing$, let $x, y \in E$, so that $f(x)=h(y)$. We have $f(x)=h(y) \in I_{f} \cap I_{h}=I_{f} \cap I_{g}$, then $f(x)=g(x)=h(y)$ (because $f, g$ are Co-injectives), and since $g$ and $h$ are Co-injectives then $x=y$. So $\forall x, y \in E, \quad f(x)=h(y) \Rightarrow x=y$. Therefore $f, h$ are Co-injectives. 


\section{Acknowledgements}

I would like to thank my dear friends, especially Chafik Bouazzaoui for us discussing the Cantor-Bernstein theorem and Mai Mohammed mainly who for his help with translating this document.

\section{Conflicts of Interest}

The author declares no conflicts of interest regarding the publication of this paper.

\section{References}

[1] Berhuy, G. (2002) Algébre: le grand combat. Clavage et Mounet, Montrouge.

[2] Dehornoy, P. (2017) La théorie des ensembles. Clavage et Mounet, Montrouge.

[3] Gourdon, X. (1994) Les maths en tête Algèbre. Ellipses, Paris.

[4] Jacobson, N. (2009) Basic Algebra I. Dover Publication Inc., New York.

[5] Guégand, T.D.J. (1999) Algèbre en classe préparatoires MP*. Ellipses, Paris. 